\title{
THE KEPLER CLUSTER STUDY: STELLAR ROTATION IN NGC 6811
}

\author{
Søren Meibom¹, Sydney A. Barnes ${ }^{2}$, David W. Latham ${ }^{1}$, Natalie Batalha ${ }^{3}$, William J. \\ Borucki $^{3}$, David G. Koch ${ }^{3}$, Gibor Basri ${ }^{4}$, Lucianne M. Walkowicz ${ }^{4}$, Kenneth A. Janes ${ }^{5}$, Jon \\ Jenkins ${ }^{6,3}$, Jeffrey Van Cleve ${ }^{6,3}$, Michael R. Haas ${ }^{3}$, Stephen T. Bryson ${ }^{3}$, Andrea K. \\ Dupree $^{1}$, Gabor Furesz ${ }^{1}$, Andrew H. Szentgyorgyi ${ }^{1}$, Lars A. Buchhave ${ }^{7,1}$, Bruce D. Clarke ${ }^{6}$, \\ Joseph D. Twicken ${ }^{6}$, Elisa V. Quintana ${ }^{6}$
}

\begin{abstract}
We present rotation periods for 71 single dwarf members of the open cluster NGC 6811 determined using photometry from NASA's Kepler Mission. The results are the first from The Kepler Cluster Study which combine Kepler's photometry with ground-based spectroscopy for cluster membership and binarity. The rotation periods delineate a tight sequence in the NGC6811 color-period diagram from $\sim 1$ day at mid-F to $\sim 11$ days at early-K spectral type. This result extends to $\sim 1$ Gyr similar prior results in the $\sim 600$ Myr Hyades and Praesepe clusters, suggesting that rotation periods for cool dwarf stars delineate a welldefined surface in the 3-dimensional space of color (mass), rotation, and age. It implies that reliable ages can be derived for field dwarf stars with measured colors and rotation periods, and it promises to enable further understanding of various aspects of stellar rotation and activity for cool stars.
\end{abstract}

Subject headings: Stars: activity — Stars: ages — Stars: late-type — Stars: rotation, starspots — open clusters and associations: individual (NGC6811)

\footnotetext{
${ }^{1}$ Harvard-Smithsonian Center for Astrophysics, Cambridge, MA, 02138, USA

${ }^{2}$ Lowell Observatory, Flagstaff, AZ, 86001, USA

${ }^{3}$ NASA Ames Research Center, Moffett Field, CA 94035, USA

${ }^{4}$ Astronomy Department, University of California, Berkeley, CA 94720, USA

${ }^{5}$ Department of Astronomy, Boston University, Boston, MA 02215, USA

${ }^{6}$ SETI Institute, Mountain View, CA 94043, USA

${ }^{7}$ Niels Bohr Institute, Copenhagen University, Denmark
} 


\section{INTRODUCTION}

Cool stars lose angular momentum and spin down with time. Observations indicate that the surface rotation period $P$, of a cool main sequence starl 1 is mainly dependent on its age $t$, and mass $M ; P=P(t, M)$. These dual dependencies suggest that a surface exists in the $P-t-M$ space which can be defined from measurements of the colors (masses) and periods for stars with known ages. Observations to define the $P-t-M$ surface will simultaneously provide the dependence of $P$ on $M$ at a given $t$ (cross section across $t$-axis) and the dependence of $P$ on $t$ for a given $M$ (cross section across $M$-axis). These mass- and age-dependencies of stellar spin-down will enable a more detailed understanding of the physical processes behind the loss of angular momentum in cool stars (e.g. Kawaler 1988; Pinsonneault et al. 1990; Soderblom et al. 1993b; Collier Cameron \& Li 1994; Bouvier et al. 1997; Denissenkov et al. 2010; Barnes \& Kim 2010). The existence of a well-defined surface in $P-t-M$ space will also imply that the measurement of two of these variables yields the third. Of the three variables, $P$ and $M$ (or a suitable proxy such as color) are the easiest to measure, providing access to the stellar age $(t)$, invaluable for the chronological arrangement of stars and their companions (including planets) and thus for our understanding of various related astrophysical phenomena. The thickness of the $P-t-M$ surface will determine how well the theory of angular momentum evolution can be constrained and how precisely ages can be determined.

Rotation period measurements in young open clusters are steadily subsuming earlier $v \sin i$ data (e.g. Stauffer \& Hartmann 1987; Soderblom et al. 1993a; Jones et al. 1996; Soderblom et al. 2001; Terndrup et al. 2000, 2002) and are helping to improve the definition of $P(t, M)$. For stars in young $(\sim 100 \mathrm{Myr})$ clusters the relationship between $P$ and $M$ is not unique, and the surface splits into two (fast and slow) branches (e.g. Barnes 2003; Hartman et al. 2009, 2010; Meibom et al. 2009, 2011; Irwin et al. 2009; James et al. 2010). With time, the fast branch dissipates as stars converge in their rotational evolution onto the slow branch, resulting in a unique relationship between $P$ and $M$ at $\sim 600$ Myr (Radick et al. 1987; Collier Cameron et al. 2009; Delorme et al. 2011). The Hyades and Praesepe clusters constitute the oldest coeval stellar populations for which rotation periods have been measured. Older stars rotate more slowly and are less active, and the lack of periods in clusters older than Hyades/Praesepe reflects the challenging task of measuring - from the ground the spot-induced photometric fluctuations required to derive rotation periods. Consequently, the shape and position of the $P-t-M$ surface for $t \gtrsim 600 \mathrm{Myr}$ is constrained only by the rotation period, age, and mass of a single star - the Sun. (Ages of field stars with measured periods are too imprecisely known to specify the surface.)

\footnotetext{
${ }^{1}$ With the exception of stars in systems where tidal effects have influenced the rotation rate.
} 
NASA's Kepler mission offers a special opportunity to overcome this difficulty. Kepler provides nearly uninterrupted photometric measurements of unprecedented duration (years), cadence (minutes), and precision ( $p p m)$. As part of the Kepler mission, The Kepler Cluster Study (Meibom \& Kepler Team 2010) is targeting three open clusters older than the Hyades with the goal of deriving rotation periods for their cool main-sequence members. If successful, this effort will dramatically improve our empirical understanding of the spin-down rates of low-mass stars of different masses by verifying the existence and precisely defining the shape of the $P-t-M$ surface beyond the age of the Hyades and possibly that of the Sun. In this paper we report the first results from the Kepler Cluster Study for the $~ 1$ Gyr cluster NGC 6811. The results confirm the existence of a unique surface out to the age of NGC 6811, and specify its shape, $P(M)$, at that age. In future papers, we will extend $P(t, M)$ to $t=2.5 \mathrm{Gyr}$ (NGC 6819) and possibly $t=9$ Gyr (NGC 6791).

\section{THE KEPLER CLUSTER STUDY}

Figure 1 demonstrates the current severely uneven observational coverage of the $P$ $t-M$ parameter-space. The orange surface represents an extrapolation in age, using the Skumanich $P \propto \sqrt{t}$ spin-down law (Skumanich 1972), of the color-period relation observed among moderate-to-slow rotators in the Hyades and younger clusters (black curve).

The Kepler Cluster Study is a program to identify members of the four open clusters within the Kepler field of view and to obtain and analyze Kepler light curves for those members to measure stellar rotation periods and search for transiting planets. The four clusters are NGC 6866 (0.5 Gyr), NGC 6811 (1 Gyr), NGC 6819 (2.5 Gyr), and NGC 6791 (9 Gyr). As coeval, cospatial, and chemically homogeneous collections of stars with a range of masses, for which precise ages can be determined, open clusters are the best opportunity we have for studying the dependencies of rotation on the most fundamental stellar properties - age and mass. The potential contributions to the study of stellar rotation by the Kepler Cluster Study are shown as blue dashed curves in Figure 1, The curves represent the color ranges for the main-sequence members currently being observed by Kepler in the four clusters.

Although certain information about NGC 6811 is already available (Sanders 1971; Lindoff 1972; Barkhatova et al. 1978; Glushkova et al. 1999; Mills et al. 2005; Luo et al. 2009), it was not particularly well-studied prior to its inclusion in the Kepler Cluster Study. Consequently, even basic properties of the cluster are uncertain or unknown. Located near Cygnus and Lyra $\left(\alpha_{2000}=19^{h} 37 m, \delta_{2000}=+46^{\circ} 23^{\prime} ; l=79.2, b=12^{\circ} .0\right)$, its color-magnitude diagram (CMD) is highly contaminated with field stars (see Figure 2), making an extensive groundbased radial-velocity survey essential to identify cluster members and to improve cluster 


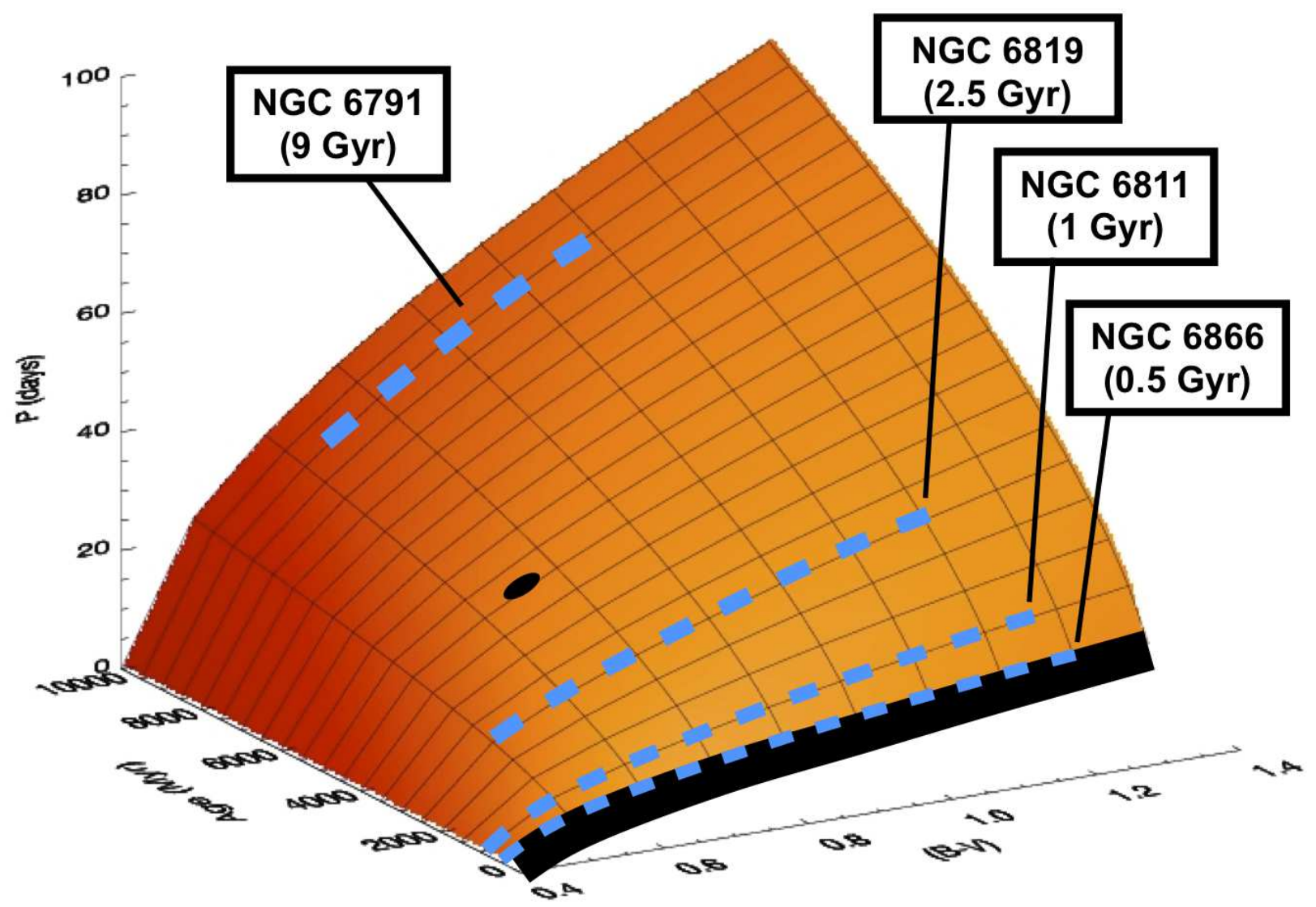

Fig. 1.- A surface (orange) in the 3-dimensional space of color (mass, x-axis), age (Myr, yaxis), and stellar rotation period (Days, z-axis). The surface is an extrapolation in age, using $P \propto \sqrt{t}$ (Skumanich 1972), of the color-period relation observed among moderate-to-slow rotators in the Hyades and younger clusters (black curve). The black dot marks the color, age, and rotation period of the Sun. The dashed blue curves mark the ages and color-ranges of the stars being observed by Kepler in the four open clusters located within its field of view.

parameters. The $1 \mathrm{Gyr}$ cluster age quoted in this study is based on the recent photometric study by Mills et al. (2005) who found an age of $975 \mathrm{Myr}$, and on an estimate of the cluster age of $1.1 \pm 0.2 \mathrm{Gyr}$ based on the color difference between the main sequence turnoff and the red giant clump (Janes \& Hod 2011). The latter technique is independent of the cluster reddening and only moderately sensitive to the cluster metallicity. The current uncertainty in the values for the reddening and metallicity of NGC 6811 are the limiting factors in a determination of its age from main-sequence and turnoff fitting in the CMD. 


\subsection{Ground-Based Spectroscopy}

We are conducting a multi-epoch radial-velocity (RV) survey over a 1-degree diameter field centered on NGC 6811 using the 6.5m MMT telescope and the Hectochelle multi-objectspectrograph (Szentgyorgyi 2011; Mink et al. 2007; Szentgyorgyi 2006; Fabricant et al. 2005). This work identifies late-type members of the cluster to be observed by Kepler. To date, $\sim 6000$ spectra have been obtained of nearly 3100 stars in the field of NGC 6811. Of these, 363 stars are members or candidate members 2 and 228 of those have so far not shown significant velocity variation and are considered spectroscopically single. These numbers underscore the high level of field star contamination ( $\sim 90 \%$ on average). Figure 2 shows the location of all single members (red dots) and the 71 members for which we have measured periods (blue asterisks) in the NGC 6811 CMD.

\section{DATA AND ANALYSIS}

Details of the Kepler mission, spacecraft, and photometer have been presented elsewhere (e.g. Borucki et al. (2010), Koch et al. (2010), Batalha et al. (2010), Caldwell et al. (2010), Gilliland et al. (2010), Jenkins et al. (2010a,b)).

We analyze four quarters 3 of Kepler data (Q1-Q4) spanning a period of 310 days from 2009 May 12 through 2010 March 20. We use Kepler data summed into long cadence bins ( $\sim 30$ minutes). Stars observed in all 4 quarters have $\sim 13,000$ flux measurements. Not all stars studied were observed in all 4 quarters. Each stellar flux measurement is the result of simple aperture photometry using an aperture which optimizes the signal-to-noise ratio. The spectral response of the Kepler bandpass $(423-897 \mathrm{~nm})$ is similar to broadband $\mathrm{V}+\mathrm{R}$.

The stellar rotation periods presented in this paper are based on analysis of light curves resulting from the Kepler data analysis pipeline and corrected by the Pre-search Data Conditioning (PDC) routine. In this process aperture photometry is performed on calibrated pixel data with the sky signal removed. In the PDC process, attempts are made to remove signals in the data from pointing drifts, focus changes, and thermal variations. As described in the Kepler Data Release Notes (Van Cleve et al. 2010), the pipeline is still under development and is primarily intended to optimize the search for planetary transits. Accordingly, the pro-

\footnotetext{
${ }^{2}$ Stars with less than 4 RV measurements are considered candidate members until additional measurements confirm their membership.

${ }^{3}$ The period of time between rotation of the spacecraft around the optical axis to re-orient its solar panels and radiator.
} 


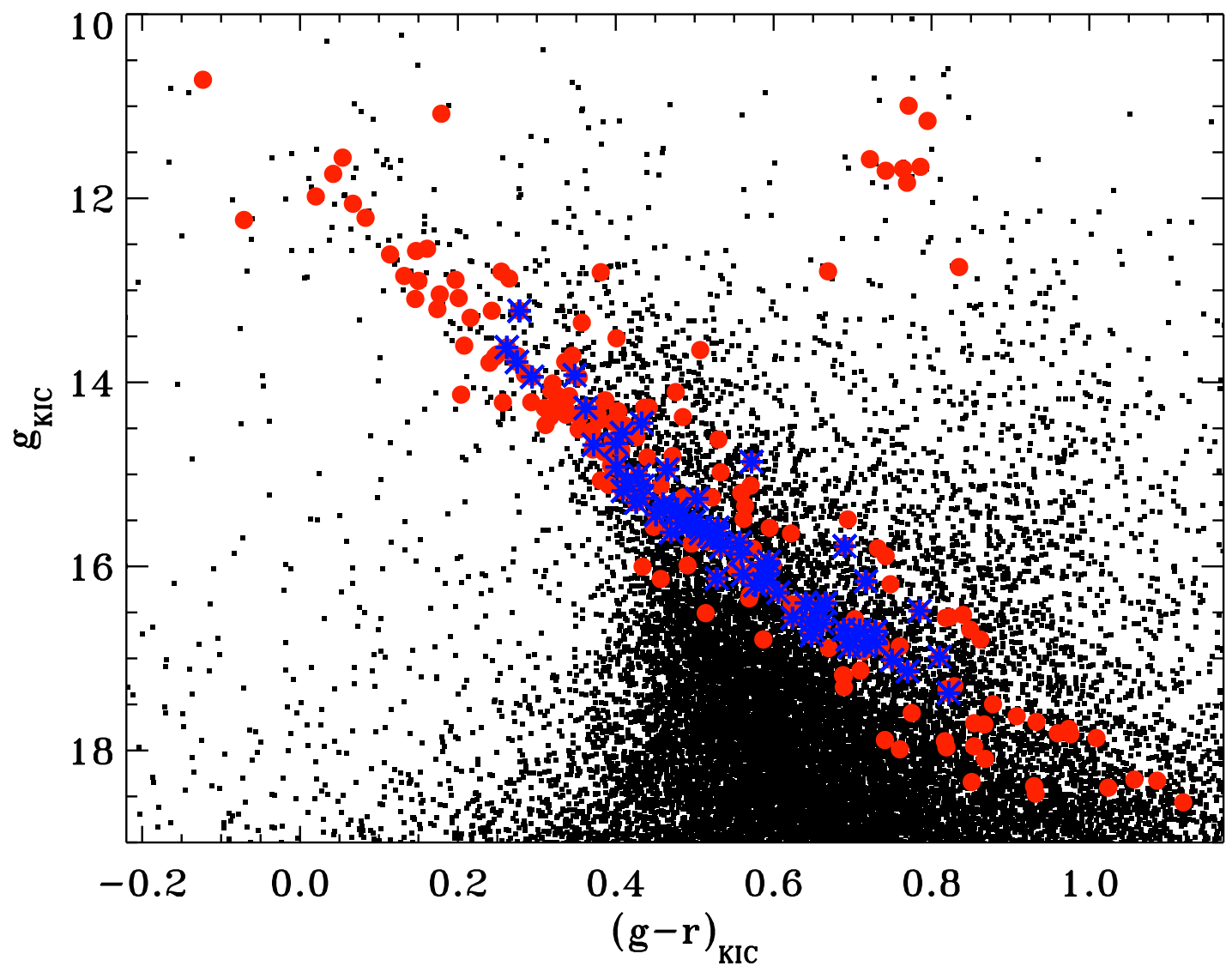

Fig. 2.- The color-magnitude diagram of NGC6811. Photometry is in the SDSS $g$ and $r$ bands and from the Kepler Input Catalog (KIC; Brown et al. 2011, http://archive.stsci.edu/kepler). The diagram shows all stars in the KIC within a 0.5-degree radius of the cluster center. Spectroscopically single RV members and candidate members are marked in red. The 71 members for which we present rotation periods are marked with blue asterisks.

cessing may not preserve all stellar variability with amplitudes comparable to or smaller than the instrumental systematics on long timescales. However, the rotation periods presented in this study are derived from photometric variability with amplitudes (typically 1-3\%) greater and time-scales shorter than relevant uncorrected instrumental and data processing systematics.

Prior to our period search, all quarters of data were normalized by the median signal and stitched together to form a single light curve for each star. We employed the Scargle 
(1982) periodogram analysis to detect periodic variability. We searched 20,000 frequencies corresponding to periods between 0.05 day and 100 days. The rotation period for a given star was determined from 2-6 time-intervals distributed over the four quarters. These intervals were selected to avoid and minimize the effect on the measured period from multiple spot groups and trends not removed by the data processing. For all periods reported we have examined - by eye - the periodogram and raw and phased light curves. We have also checked the periods independently using the CLEAN algorithm of Roberts et al. (1987). Figure 3 shows the normalized Aperture photometry Corrected Flux (ACF) for a single member of NGC 6811. In addition to the clear modulations due to stellar spots, low frequency trends are visible for each quarter as well as discontinuities between quarters. The five intervals selected for measurements of the rotation period are shaded grey.

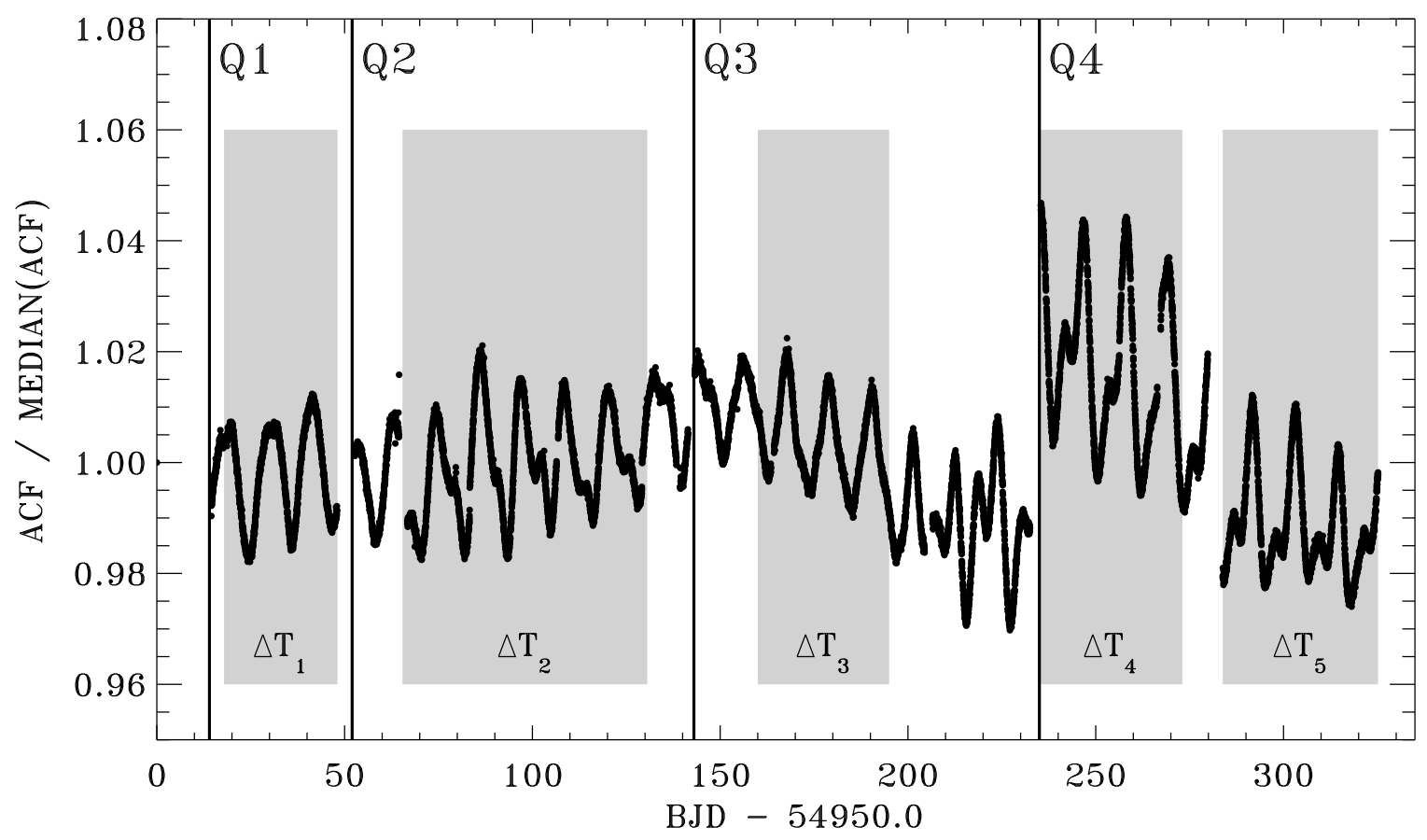

Fig. 3.- Kepler light curve for a single member (Kepler ID: 9531467) of NGC 6811 showing long cadence data from Q1-Q4. The beginning of each quarter is marked and labeled. The flux time-series for each quarter is normalized to the median flux in the respective quarter. Grey boxes mark the time-intervals $\left(\Delta T_{1}, \Delta T_{2}, \Delta T_{3}, \Delta T_{4}\right.$, and $\left.\Delta T_{5}\right)$ used for period determination for this particular star. 


\section{RESULTS AND IMPLICATIONS}

The left panel of Figure 4 displays our color-period diagram for NGC 6811. Rotation periods for the 71 candidate single members identified from the spectroscopic survey are displayed against their $g-r$ colors. We observe that, apart from a few outliers (discussed below), the distribution of periods forms a single and narrow rotational sequence from mid$\mathrm{F}$ through early-K spectral type. The rotation periods are $\sim 1-2$ days for $g-r<0.4$, and rise sharply at $g-r \sim 0.4$ towards $\sim 11$ days for the redder stars. Three stars deviate significantly from the NGC 6811 sequence. Kepler IDs $9717386(P=16.4, g-r=0.62), 9595724(P=$ 12.7, $g-r=0.47)$, and $9469799(P=2.5, g-r=0.57)$. All three stars are candidate single members of NGC 6811 with 2 RV measurements and 3-4 measurements of their rotation periods over Q1-Q4. With only 2 RV measurements their membership and/or their single star status can change with further observations. Table 1 shows a stub version of a table available online with Kepler IDs, astrometry, photometry, rotation periods, and RV membership status for all 71 stars.

To test for non-astrophysical periodic signals in the Kepler photometry introduced by either the instrument or by the processing pipeline, we analyzed the light curves for 100 randomly selected field dwarfs in the NGC6811 field. We found no dominant frequencies and only rarely $(<5 \%)$ periodic photometric variability with an amplitude similar to those observed for members of NGC 6811 for which we derived rotation periods.

The sequence in the NGC 6811 color-period diagram is equivalent to the sequences observed for FGK dwarfs in the Hyades (Radick et al. 1987; Delorme et al. 2011), Praesepe (Delorme et al. 2011), Coma Berenices (Collier Cameron et al. 2009), and M 37 (Hartman et al. 2009), and to the slow sequences seen in numerous younger open clusters for the same spectral range. Barnes (2003) labeled it the $I$ sequence. A second sequence of ultra fast rotators

Table 1. Data for the 71 members of NGC 6811 with measured rotation periods.

\begin{tabular}{|c|c|c|c|c|c|c|c|c|}
\hline $\mathrm{Ke}$ & $\begin{array}{c}\text { RA } \\
\left(\begin{array}{lll}h & m & s\end{array}\right)\end{array}$ & $\begin{array}{c}\text { DEC } \\
\left(\begin{array}{lll}\circ & \prime & \prime \prime\end{array}\right)\end{array}$ & $g$ & $r$ & $\begin{array}{c}P \\
\text { (Days) }\end{array}$ & $\begin{array}{c}\sigma_{P} \\
\text { (Days) }\end{array}$ & $N_{P}$ & \\
\hline & 193639.33 & 462701.83 & & & & 0.01 & 5 & \\
\hline 9594100 & 193655 . & 461518. & 13 & 12 & 6 & .01 & 6 & CSM \\
\hline 9716563 & 193738.57 & 462912.68 & 13.92 & 13.57 & 1.29 & 0.21 & 4 & $\mathrm{SM}$ \\
\hline 9716817 & 193759.91 & 462444.75 & 14.55 & 14.14 & 1.36 & 0.01 & 4 & $\mathrm{SM}$ \\
\hline 9654924 & 193641.17 & 462309.52 & 13.94 & 13.65 & 1.60 & 0.22 & 4 & CSM \\
\hline
\end{tabular}


with periods of $\leq 1$ day found in the youngest $(\sim 100 \mathrm{Myr})$ open clusters [e.g. the Pleiades (van Leeuwen \& Alphenaar 1982; Hartman et al. 2010) and M 35 (Meibom et al. 2009)], is absent in NGC 6811 as it is in the Hyades and Coma.
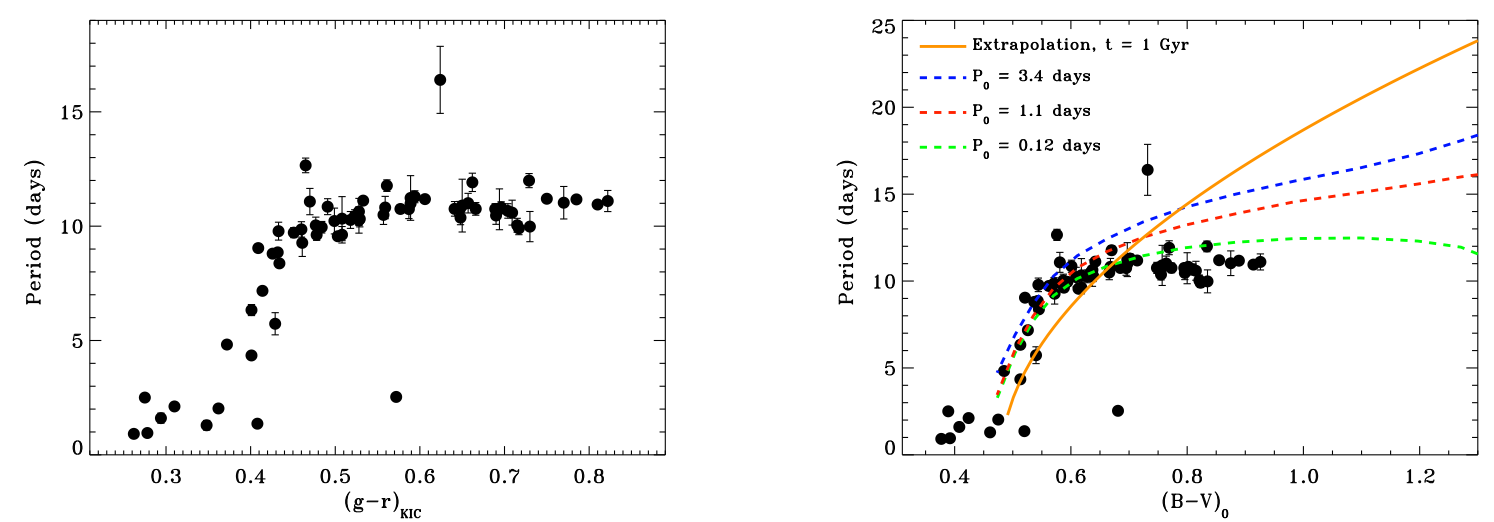

Fig. 4.- Left: The observed color-period diagram in $g-r$ color for 71 FGK candidate single members of NGC 6811. The periods define a clear $I$-type sequence. The error-bars represent the RMS of multiple period measurements. The sequence is the observed representation of a cross-section at the age of NGC6811 $(P=P(1 \mathrm{Gyr}, M))$ of the surface $P=P(t, M)$. Right: The color-period diagram in de-reddened $B-V$ color using $E_{(B-V)}=0.1$. The orange curve represents the simple extrapolation to $1 \mathrm{Gyr}$, using the Skumanich $P \propto \sqrt{t}$ law, of the color-period relation from younger open clusters. The green, blue, and red curves are rotational isochrones for $t=1 \mathrm{Gyr}$ calculated using the rotational evolution theory described in Barnes (2010), and correspond to initial (ZAMS) periods of 0.12 days, 1.1 days, and 3.4 days respectively.

The most basic implication of the NGC 6811 color-period diagram is that the relatively tight sequence of rotators seen in the Hyades, Coma Berenices, Praesepe, and M 37 continues to remain tight at later ages, and extends to at least 1 Gyr. Because the rotational evolution of (single) cool stars is convergent, we expect that older clusters will also display similarly tight sequences. The totality of such data therefore will define the desired $P-t-M$ surface. Although based on preliminary analysis of the spectroscopic and photometric data, the NGC 6811 color-period diagram constitutes a proof of concept for the Kepler Cluster Study, and represents a significant increase in our empirical knowledge of how cool stars spin down.

The dependence of $P$ on color (or equivalently $M$ ) defined by the NGC $6811 I$ sequence is a cross-section at $t \simeq 1$ Gyr of the hypothesized $P$ - $t$ - $M$ surface. The scatter along the sequence is consistent with the measurement uncertainty (and differential rotation) for individual stars and confirms that the surface is intrinsically thin. This has important implications 
for its application to determine stellar ages from measurements of colors and rotation periods (gyrochronology, Barnes 2003, 2007; Mamajek \& Hillenbrand 2008; Soderblom 2010). The thinner the surface the more precisely a gyrochronology age of a star can be determined.

Knowing stellar ages is fundamental to understanding the time-evolution of various astronomical phenomena related to stars and their companions. For the vast majority of stars not in clusters (unevolved late-type field stars including most exoplanet host stars), ages determined using the isochrone method are highly uncertain because the primary ageindicators are nearly constant throughout their main-sequence lifetimes, and because their distances and thus luminosities are poorly known. For such stars rotation could serve as a superior age indicator. The tight rotational sequence in NGC 6811 will serve to further develop the technique of gyrochronology and thereby enable better ages for unevolved latetype field stars with measured periods.

Finally, we note that these results provide new and valuable constraints for models of angular momentum evolution. The right panel of Figure 4 displays the color-period diagram in de-reddened $B-V$ color [transformed from $g-r$ using equations by Jester et al. (2005) and corrected by $E_{(B-V)}=0.1$ ] with the empirical color-period relation from younger clusters extrapolated to 1 Gyr using $P \propto \sqrt{t}$ (cross-section of orange surface in Figure 1) and with rotational evolution models from Barnes (2010). The green, blue, and red theoretical curves correspond to the range of initial periods allowed on the Zero Age Main Sequence (ZAMS). While preliminary, the comparison suggests that simple extrapolation, using the Skumanich $\sqrt{t}$ law, of the color-period relation from younger clusters cannot account for the shape of the NGC 6811 sequence. We emphasize that the current value for the cluster reddening is uncertain, but even with agreement for late-F and early-G dwarfs, the extrapolated and theoretical periods are longer than observed for the mid-G to early-K dwarfs. Additional work is envisioned to understand this and other related issues in cool star angular momentum evolution.

Further analysis of ground-based spectroscopic and photometric data will enable us to improve our knowledge of cluster properties such as age, distance, and metallicity. In addition to our ongoing spectroscopic survey of the cluster we have acquired new groundbased photometric data in UBVI filters, and will use these to provided improved photometric information and reddening for the cluster in due course. 


\section{CONCLUSIONS}

We present the first results from the Kepler Cluster Study for the open cluster NGC 6811. With rotation periods measured for 71 radial-velocity and photometric cluster members, the NGC 6811 color-period diagram displays a single tight rotational sequence from mid-F to early-K spectral type. This result extends to $\sim 1$ Gyr similar sequences observed between 550-600 Myr in the Hyades, Praesepe, Coma Berenices, and M37, and suggests that cool stars populate a thin surface in rotation-age-mass space. The result will enable a more detailed understanding of the rotational evolution of cool stars and implies that credible ages can be derived for late-type dwarfs with measured colors and rotation periods.

Kepler was competitively selected as the tenth Discovery mission. Funding for this mission is provided by NASA's Science Mission Directorate. We thank the entire Kepler Mission team including engineers, managers, and administrative staff, who have all contributed to the success of the mission. This work has been supported by NASA grant NNX09AH18A (The Kepler Cluster Study) to S.M., and by support to S.M. from the Kepler mission via NASA Cooperative Agreement NCC2-1390. This paper uses data products produced by the

Optical and Infrared (OIR) Astronomy Division's Telescope Data Center, supported by the Smithsonian Astrophysical Observatory. S.M. express deep appreciation for time awarded on MMT/Hectochelle and for the exceptional and friendly support of the MMT staff. The Kepler data presented in this paper were obtained from the Multimission Archive at the Space Telescope Science Institute (MAST). STScI is operated by the Association of Universities for Research in Astronomy, Inc., under NASA contract NAS5-26555. Support for MAST for non-HST data is provided by the NASA Office of Space Science via grant NNX09AF08G and by other grants and contracts.

Facilities: NASA Kepler, MMT (Hectochelle).

\section{REFERENCES}

Barkhatova, K. A., Zakharova, P. E., \& Shashkina, L. P. 1978, Soviet Ast., 22, 31

Barnes, S. A. 2003, ApJ, 586, 464

-. 2007, ApJ, 669, 1167

—. 2010, ApJ, 722, 222

Barnes, S. A., \& Kim, Y. 2010, ApJ, 721, 675 
Bouvier, J., Forestini, M., \& Allain, S. 1997, A\&A, 326, 1023

Brown, T. M., Latham, D. W., Everett, M. E., \& Esquerdo, G. A. 2011, ArXiv e-prints

Collier Cameron, A., Davidson, V. A., Hebb, L., Skinner, G., Anderson, D. R., Christian, D. J., Clarkson, W. I., Enoch, B., Irwin, J., Joshi, Y., Haswell, C. A., Hellier, C., Horne, K. D., Kane, S. R., Lister, T. A., Maxted, P. F. L., Norton, A. J., Parley, N., Pollacco, D., Ryans, R., Scholz, A., Skillen, I., Smalley, B., Street, R. A., West, R. G., Wilson, D. M., \& Wheatley, P. J. 2009, MNRAS, 1358

Collier Cameron, A., \& Li, J. 1994, MNRAS, 269, 1099

Delorme, P., Collier Cameron, A., Hebb, L., Rostron, J., Lister, T. A., Norton, A. J., Pollacco, D., \& West, R. G. 2011, ArXiv e-prints

Denissenkov, P. A., Pinsonneault, M., Terndrup, D. M., \& Newsham, G. 2010, ApJ, 716, 1269

Fabricant, D., Fata, R., Roll, J., Hertz, E., Caldwell, N., Gauron, T., Geary, J., McLeod, B., Szentgyorgyi, A., Zajac, J., Kurtz, M., Barberis, J., Bergner, H., Brown, W., Conroy, M., Eng, R., Geller, M., Goddard, R., Honsa, M., Mueller, M., Mink, D., Ordway, M., Tokarz, S., Woods, D., Wyatt, W., Epps, H., \& Dell'Antonio, I. 2005, PASP, 117, 1411

Glushkova, E. V., Batyrshinova, V. M., \& Ibragimov, M. A. 1999, Astronomy Letters, 25, 86

Hartman, J. D., Bakos, G. Á., Kovács, G., \& Noyes, R. W. 2010, ArXiv e-prints

Hartman, J. D., Gaudi, B. S., Pinsonneault, M. H., Stanek, K. Z., Holman, M. J., McLeod, B. A., Meibom, S., Barranco, J. A., \& Kalirai, J. S. 2009, ApJ, 691, 342

Irwin, J., Aigrain, S., Bouvier, J., Hebb, L., Hodgkin, S., Irwin, M., \& Moraux, E. 2009, MNRAS, 392, 1456

James, D. J., Barnes, S. A., Meibom, S., Lockwood, G. W., Levine, S. E., Deliyannis, C., Platais, I., Steinhauer, A., \& Hurley, B. K. 2010, A\&A, 515, A100+

Janes, K. A., \& Hoq, S. 2011, AJ, 141, 92

Jester, S., Schneider, D. P., Richards, G. T., Green, R. F., Schmidt, M., Hall, P. B., Strauss, M. A., Vanden Berk, D. E., Stoughton, C., Gunn, J. E., Brinkmann, J., Kent, S. M., Smith, J. A., Tucker, D. L., \& Yanny, B. 2005, AJ, 130, 873 
Jones, B. F., Fischer, D. A., \& Stauffer, J. R. 1996, AJ, 112, 1562

Kawaler, S. D. 1988, ApJ, 333, 236

Lindoff, U. 1972, A\&A, 16, 315

Luo, Y. P., Zhang, X. B., Luo, C. Q., Deng, L. C., \& Luo, Z. Q. 2009, New A, 14, 584

Mamajek, E. E., \& Hillenbrand, L. A. 2008, ApJ, 687, 1264

Meibom, S., \& Kepler Team. 2010, in Bulletin of the American Astronomical Society, Vol. 42, $284-+$

Meibom, S., Mathieu, R. D., \& Stassun, K. G. 2009, ApJ, 695, 679

Meibom, S., Mathieu, R. D., Stassun, K. G., Liebesny, P., \& Saar, S. H. 2011, Accepted to The Astrophysical Journal

Mills, E. A., Deliyannis, C. P., Sarajedini, A., \& Platais, I. 2005, in Bulletin of the American Astronomical Society, Vol. 37, Bulletin of the American Astronomical Society, 1278-+

Mink, D. J., Wyatt, W. F., Caldwell, N., Conroy, M. A., Furesz, G., \& Tokarz, S. P. 2007, in Astronomical Society of the Pacific Conference Series, Vol. 376, Astronomical Data Analysis Software and Systems XVI, ed. R. A. Shaw, F. Hill, \& D. J. Bell, 249-+

Pinsonneault, M. H., Kawaler, S. D., \& Demarque, P. 1990, ApJS, 74, 501

Radick, R. R., Thompson, D. T., Lockwood, G. W., Duncan, D. K., \& Baggett, W. E. 1987, ApJ, 321, 459

Roberts, D. H., Lehar, J., \& Dreher, J. W. 1987, AJ, 93, 968

Sanders, W. L. 1971, A\&A, 15, 368

Scargle, J. D. 1982, ApJ, 263, 835

Skumanich, A. 1972, ApJ, 171, 565

Soderblom, D. R. 2010, ARA\&A, 48, 581

Soderblom, D. R., Jones, B. F., \& Fischer, D. 2001, ApJ, 563, 334

Soderblom, D. R., Stauffer, J. R., Hudon, J. D., \& Jones, B. F. 1993a, ApJS, 85, 315

Soderblom, D. R., Stauffer, J. R., MacGregor, K. B., \& Jones, B. F. 1993b, ApJ, 409, 624 
Stauffer, J. R., \& Hartmann, L. W. 1987, ApJ, 318, 337

Szentgyorgyi, A. 2006, New A Rev., 50, 326

Szentgyorgyi, A. e. a. 2011, PASP

Terndrup, D. M., Pinsonneault, M., Jeffries, R. D., Ford, A., Stauffer, J. R., \& Sills, A. 2002, ApJ, 576, 950

Terndrup, D. M., Stauffer, J. R., Pinsonneault, M. H., Sills, A., Yuan, Y., Jones, B. F., Fischer, D., \& Krishnamurthi, A. 2000, AJ, 119, 1303

Van Cleve, J., Christiansen, J., \& Machalek, P. 2010, http://archive.stsci.edu/kepler/documents.html

van Leeuwen, F., \& Alphenaar, P. 1982, The Messenger, 28, 15 